UT-06-09

\title{
The Polonyi Problem and Upper bound on Inflation Scale in Supergravity
}

\author{
M. Ibe ${ }^{1}$, Y. Shinbara ${ }^{1}$ and T.T. Yanagida ${ }^{1,2}$ \\ ${ }^{1}$ Department of Physics, University of Tokyo, \\ Tokyo 113-0033, Japan \\ ${ }^{2}$ Research Center for the Early Universe, University of Tokyo, \\ Tokyo 113-0033, Japan
}

\begin{abstract}
We reconsider the Polonyi problem in gravity-mediation models for supersymmetry (SUSY) breaking. It has been argued that there is no problem in the dynamical SUSY breaking scenarios, since the Polonyi field acquires a sufficiently large mass of the order of the dynamical SUSY-breaking scale $\Lambda_{\mathrm{SUSY}}$. However, we find that a linear term of the Polonyi field in the Kähler potential brings us back to the Polonyi problem, unless the inflation scale is sufficiently low, $H_{\text {inf }} \lesssim 10^{8} \mathrm{GeV}$, or the reheating temperature is extremely low, $T_{R} \lesssim 100 \mathrm{GeV}$. Here, this Polonyi problem is more serious than the original one, since the Polonyi field mainly decays into a pair of gravitinos.
\end{abstract}




\section{Introduction}

In gravity-mediation models for supersymmetry (SUSY) breaking, gaugino masses in the SUSY standard model (SSM) are given by a singlet field F-term in a hidden sector. This singlet field $S$ called as Polonyi field should be an elementary field, since the gaugino masses are suppressed by higher powers of the Planck scale $M_{G} \simeq 2.4 \times 10^{18} \mathrm{GeV}$, otherwise. This singlet field is completely neutral of any symmetry and the origin of the field has no enhanced symmetry. Thus, the minima of its potential during inflation and at the true vacuum are different from each other. The distance between those minima is likely of the order of the Planck scale, $\Delta S \simeq \mathcal{O}\left(M_{G}\right)$. Therefore, after the inflation the Polonyi $S$ field starts a coherent oscillation around the true minimum when the Hubble parameter becomes of the order of the mass of $S$ and its energy density dominates the early universe if its lifetime is much longer than that of the inflaton. As long as there is no physical scale besides the Planck scale at high energies, the Polonyi field has a mass of the order of the gravitino mass $m_{3 / 2}=\mathcal{O}(1) \mathrm{TeV}$ and it decays to the SSM particles at very late times. The energetic photons and hadrons produced by the decay deconstruct the light nuclei created by the big-bang nucleosynthesis (BBN). This is called "the Polonyi problem" [1].

Possible solutions to the above problem may be found if one introduces a new high energy scale $M_{*}$ besides the $M_{G}\left(M_{*}<M_{G}\right)$. There are two possibilities to use this new scale. One is to increase interactions between hidden and observed sectors to make the decay of $S$ faster, for instance, $K=\left(1 / M_{*}\right) S^{\dagger} q^{\dagger} q+$ h.c. in a Kähler potential. Here, $q$ denotes quarks in the SSM. However, such interactions also increase the gaugino masses and one should decrease the gravitino mass by a factor $M_{*} / M_{G}$ to keep the gaugino masses at the order of $1 \mathrm{TeV}$. Then, the Polonyi mass is reduced also by the same factor, which results in even worse situation. The second possibility is to increase the interactions among fields in the hidden sector. Namely, one introduces $K=\left(1 / M_{*}^{2}\right)\left(S^{\dagger} S\right)^{2}$ for instance. Then, the mass of the Polonyi field becomes larger than the gravitino mass $m_{3 / 2}$ and the $S$ can decay before the BBN.

However, even in the second possibility, there arises another kind of Polonyi problem. The Polonyi field is much heavier than the gravitino and hence it decays mainly into a pair of gravitinos which, in turn, results in "the gravitino overproduction problem" [2]. Thus, there is still a severe upper bound on the relic abundance of the Polonyi field. (Notice that, even if one increases the interactions with the SSM particles as above, as well as the mass of the Polonyi field, the decay into gravitino is still a dominant decay mode of the Polonyi field.) 
Despite the above gravitino overproduction problem, the second possibility is very interesting. The presence of the new cut-off scale $M_{*}$ will stop $S$ to run away from the origin and the distance between the potential-minimum points of $S$ during the inflation and $S$ at the true vacuum becomes of the order of $M_{*}$, that is $\Delta S \simeq \mathcal{O}\left(M_{*}\right)$. Hence, the coherent oscillation of the Polonyi field $S$ does not necessarily dominate the universe, if $M_{*}$ is sufficiently smaller than the Planck scale $M_{G}$. Thus, as shown in the next section, the gravitino overproduction problem from the Polonyi decay can be evaded for small values of the new scale $M_{*}$ as $M_{*} \lesssim 10^{12-13} \mathrm{GeV}$. This result strongly suggests that the new scale is nothing but the dynamical scale of the SUSY breaking.

The purpose of this letter is to examine if the Polonyi problem is solved when SUSY is dynamically broken by some strong gauge interactions. In the above argument, we have assumed $\Delta S \simeq \mathcal{O}\left(M_{*}\right)$. However, we find that it is not always the case, since the potential of the $S$ is flatter than the mass term $m_{s}^{2}|S|^{2}$ above $S \simeq M_{*}$. Thus, a careful analysis on the potential of $S$ is required during the inflation. We show as a result that there is a stringent constraint on the Hubble parameter of the inflation or on the reheating temperature for a successful solution, that is, $H_{\mathrm{inf}} \lesssim 10^{8} \mathrm{GeV}$ or $T_{R} \lesssim 10^{2} \mathrm{GeV}$. This concludes that the gravity-mediation models favor relatively low-energy scale inflations as realized naturally in new inflation models. We consider that the present conclusion is quite generic, although we derive it in a class of SUSY-breaking models.

\section{Upper bound on the new scale $M_{*}$}

Before going to discuss the dynamical SUSY-breaking models, we show that there is an upper bound on the new energy scale $M_{*}$. As we have discussed in Introduction, we assume, for a moment, that the presence of the new scale $M_{*}$ may set the distance between minimum points of $S$ during the inflation and $S$ at the true vacuum to the order of $M_{*}$, that is $\Delta S \simeq M_{*}$.

After the inflation, the value of the Polonyi field is fixed at the $\Delta S \simeq M_{*}$ until the Hubble parameter $H$ falls to the mass of the Polonyi field, $H \simeq m_{s}$, and then, the Polonyi field begins a coherent oscillation around its true minimum. Here the mass of the Polonyi field is enhanced by a factor of $1 / M_{*}$ compared to the gravitino mass as discussed in Introduction, ${ }^{1}$

$$
m_{s} \simeq \frac{m_{3 / 2}}{M_{*}} .
$$

\footnotetext{
${ }^{1}$ Here and henceforth, we have taken the unit with the reduced Planck scale, $M_{G}=1$.
} 
The Polonyi field $S$ and the inflaton $\phi$ decay into radiation when the Hubble parameter becomes at the decay rate of the Polonyi field,

$$
\Gamma_{s} \simeq \frac{3}{288 \pi} \frac{m_{s}^{5}}{m_{3 / 2}^{2}}
$$

and at that of the inflaton,

$$
\Gamma_{\phi}=\left(\frac{\pi^{2} g_{*}}{90}\right)^{1 / 2} T_{R}^{2}
$$

respectively. In Eq. (2), we present a decay rate of the Polonyi field into a pair of gravitinos, since the Polonyi field mainly decays into a gravitino pair. ${ }^{2}$ In Eq. (3), we have parametrized the decay rate of the inflaton by using a reheating temperature $T_{R}$ after the inflation, and $g_{*} \simeq 200$ denotes the effective massless degrees of freedom of the SSM. In the following discussion, we assume that the Polonyi field decays fast enough not to dominate the energy density of the universe after the inflaton decay.

The ratio of the number density of the gravitino to entropy is given by (after the inflaton decay),

$$
Y_{3 / 2}=\frac{n_{3 / 2}}{s} \gtrsim 2 \frac{3 T_{R}}{4 m_{\phi}} \frac{n_{s}}{n_{\phi}} B_{R}
$$

Here, we have assumed that most of the gravitinos are produced by the Polonyi decay. $n_{s}$ and $n_{\phi}$ denote the number densities of the Polonyi field and the inflaton at $H \simeq m_{s}, B_{R}=\mathcal{O}(1)$ the branching ratio of the Polonyi decay into a pair of gravitinos, and $m_{\phi}$ the mass of the inflaton. The factor $3 T_{R} / 4 m_{\phi}$ comes from the dilution of the gravitino by the entropy production of the inflaton decay. (The equality in Eq. (4) holds as long as the Polonyi field $S$ decays before its domination of the energy density.) The number density of the Polonyi field and the inflaton at $H \simeq m_{s}$ are given by (when the Polonyi field starts the oscillation),

$$
\begin{aligned}
n_{s} & \simeq m_{s}|\Delta S|^{2} \\
n_{\phi} & \simeq \frac{\rho_{\phi}}{m_{\phi}} \simeq \frac{3 H^{2}}{m_{\phi}}
\end{aligned}
$$

Thus, the yield of the gravitino can be expressed by,

$$
Y_{3 / 2} \gtrsim \frac{T_{R}}{2 m_{s}}(\Delta S)^{2} B_{R} \simeq \frac{T_{R}}{2 m_{3 / 2}} M_{*}^{3} B_{R}
$$

\footnotetext{
${ }^{2}$ Recently, it has been extensively discussed that the decay into a pair of gravitinos of the moduli fields 3 and the inflaton [4] is much enhanced by even small mixings of those fields with the Polonyi field.
} 
where we have used $\rho_{\phi}=3 H^{2} \simeq 3 m_{s}^{2}$ and Eq. (11).

To keep the success of the BBN, the gravitino abundance must satisfy [2],

$$
Y_{3 / 2} \lesssim 10^{-(14-16)}
$$

for $m_{3 / 2}=\mathcal{O}(1) \mathrm{TeV}$. ¿From Eq. (7), we find that an upper bound on the new scale $M_{*}$,

$$
M_{*} \lesssim 10^{12-13} \mathrm{GeV}\left(\frac{m_{3 / 2}}{1 \mathrm{TeV}}\right)^{1 / 3}\left(\frac{10^{6} \mathrm{GeV}}{T_{R}}\right)^{1 / 3} B_{R}{ }^{-1 / 3} .
$$

Notably, the above upper bound on $M_{*}$ is close to the scale of the dynamical SUSY breaking,

$$
\Lambda_{\mathrm{SUSY}} \simeq \sqrt{3^{1 / 2} m_{3 / 2}} \simeq 10^{11} \mathrm{GeV}\left(\frac{m_{3 / 2}}{1 \mathrm{TeV}}\right)^{1 / 2} .
$$

Therefore, the above constraint (91) strongly suggests that the new scale $M_{*}$ is nothing but the dynamical scale $\Lambda \simeq 4 \pi \Lambda_{\text {SUSY }}$ of strong interactions for the SUSY breaking, where the Polonyi field $S$ obtains its mass $m_{s} \sim m_{3 / 2} / \Lambda \simeq \Lambda_{\mathrm{SUSY}}$.

\section{Upper bound on the Inflation scale}

As we have seen in the previous section, the solution to the Polonyi problem using the new cut-off scale $M_{*}$ suggests the dynamical SUSY breaking by strong interactions. In this section, we discuss the Polonyi problem to examine if the dynamical SUSY breaking model can indeed solve the problem. As we have warned in Introduction, we cannot apply the result of the previous section directly, since the potential of $S$ is very flat above $S \simeq M_{*}$ and our assumption $\Delta S \simeq M_{*}$ is not guaranteed automatically. Thus, we have to arrange the inflaton potential to keep $\Delta S \lesssim M_{*}(\simeq \Lambda)$ during the inflation. We will show in this section that there is a stringent upper bound on the inflation scale.

\subsection{The scalar potential of a flat direction}

Before going to discuss the dynamics of the Polonyi field $S$ during the inflation, we consider the scalar potential of the Polonyi field in the dynamical SUSY breaking model [5, 6]. To see it

explicitly, we adopt a dynamical SUSY breaking model based on the SUSY SU(2) gauge theory with four fundamental fields $Q_{i}(i=1-4)$ and six singlet fields $S_{i j}=-S_{j i}(i, j=1-4)$. The tree-level superpotential is given by [6],

$$
W=\frac{\lambda_{i j}}{2} S_{i j} Q_{i} Q_{j}
$$


Here, $\lambda_{i j}$ 's denote coupling constants and we have omitted the gauge indices and the summations over $i, j$. The equations of motion for $S_{i j}, \partial W / \partial S_{i j}=0$, set $Q_{i} Q_{j}=0$, which contradict with the quantum modified constraint $\operatorname{Pf}\left(Q_{i} Q_{j}\right)=\Lambda^{4}$ [7]. Here, $\Lambda$ denotes the dynamical scale of the gauge interactions. Hence, the SUSY is broken dynamically.

In this model, there is a flat direction which is a linear combination $S$ of the singlets, $S_{i j}$, which corresponds to the Polonyi field in the previous section. For $S$ near the origin, $S \ll \Lambda / \lambda$, the superpotential Eq. (11) can be effectively written as,

$$
W_{e f f} \simeq \lambda\left(\frac{\Lambda}{4 \pi}\right)^{2} S,
$$

by means of the quantum constraint $\operatorname{Pf}(Q Q)=\Lambda^{4}$. Here, we have used a naive dimensional counting [8], and $\lambda$ denotes an appropriate linear combination of $\lambda_{i j}$. On the contrary, for large values of $S, S \gg \Lambda / \lambda$, the $Q_{i}$ 's become massive and can be integrated out. Thus, the theory exhibits a gaugino condensation which produces an effective superpotential,

$$
W_{e f f} \simeq \lambda\left(\frac{\Lambda}{4 \pi}\right)^{2} S .
$$

Therefore, the scalar potential for all range of $S$ is given by,

$$
V(S)=\left|\lambda\left(\frac{\Lambda}{4 \pi}\right)^{2}\right|^{2}
$$

and $S$ is a flat direction.

The degeneracy of the above flat direction is lifted by quantum-effects in the Kähler potential [5]. For small value of $S(S \ll \Lambda / \lambda)$ the effective Kähler potential is expected to take a form,

$$
K=|S|^{2}+\frac{\Lambda^{2}}{16 \pi^{2}}\left(-\frac{\eta}{4}\left|\frac{\lambda S}{\Lambda}\right|^{4}+\cdots\right)
$$

where $\eta$ is a real constant which we expect to be of order one, and hereafter, $\lambda$ denotes the coupling constant at the dynamical scale $\Lambda$. It leads to a mass term of $S$ as,

$$
V_{\text {loop }} \simeq\left|\lambda\left(\frac{\Lambda}{4 \pi}\right)^{2}\right|^{2}\left[1+\frac{\eta|\lambda|^{4}}{(4 \pi \Lambda)^{2}}|S|^{2}\right] .
$$

We find the mass of the Polonyi field as

$$
m_{s}^{2} \simeq \frac{\eta|\lambda|^{4}}{(4 \pi \Lambda)^{2}}\left|\lambda\left(\frac{\Lambda}{4 \pi}\right)^{2}\right|^{2} \simeq \sqrt{3} \eta \lambda\left(\frac{\lambda}{4 \pi}\right)^{4} m_{3 / 2} \gg m_{3 / 2}^{2},
$$


where we have used a definition,

$$
m_{3 / 2}=\frac{1}{\sqrt{3}}\left|\lambda\left(\frac{\Lambda}{4 \pi}\right)^{2}\right| .
$$

Note that for $\eta>0$, the mass squared of $S$ becomes positive and the vacuum expectation value $(\mathrm{VEV})$ is $\langle S\rangle=0$. On the contrary, for $\eta<0$ the mass squared of $S$ is negative and we expect $\langle S\rangle \sim \Lambda / \lambda$, since the effective potential is lifted in the large $S$ region (see Eq. (19)) [9]. In the following, we only consider the case of $\eta>0$ and $\langle S\rangle=0$ for simplicity, since the following discussion will not be changed significantly for $\eta<0$. $^{3}$

For large values of $S(S \gg \Lambda / \lambda)$, quantum corrections to the scalar potential come from the perturbative wave function renormalization factor of $S$ and the potential is given by [9] ,

$$
V_{\text {loop }} \simeq\left|\lambda\left(\frac{\Lambda}{4 \pi}\right)^{2}\right|^{2}\left[1-\int_{\ln \Lambda}^{\ln \lambda S} \frac{\lambda(\mu)^{2}}{4 \pi^{2}} d \ln \mu\right]^{-1},
$$

where $\mu$ denotes the scale of the renormalization group ${ }^{4}$ Thus, the scalar potential in Eq. (19) is much flatter than Eq. (16). The flatness of the potential for the large field value is a generic feature of any effective O'Raifeartaigh models where flat directions are lifted by the quantum corrections.

Before closing this subsection, we stress an important feature of the Polonyi field in gravitymediation models. In gravity-mediation models, the gauginos in the SSM obtain the SUSY breaking masses via direct couplings to the Polonyi field,

$$
W=\frac{S}{M_{G}} \mathcal{W}^{\alpha} \mathcal{W}_{\alpha}
$$

where $\mathcal{W}^{\alpha}$ 's denote gauge field strength chiral superfields. Hence, we expect that the Polonyi field must be neutral under any symmetries. This means that, in general, we cannot forbid a linear term of the Polonyi field in the Kähler potential,

$$
K=|S|^{2}-c^{*} S-c S^{\dagger}+\cdots
$$

where $c$ is a dimensionful parameter and is expected to be of the order of the Planck scale $M_{G}=1$. Furthermore, even if we set $c=0$ at the tree-level, the interaction terms such as Eq. (20) generate the linear term of order $c=\mathcal{O}\left(N_{g} / 16 \pi^{2}\right)$ at one loop level, where $N_{g}$ is the number of the gauge multiplets circulating in the loop diagrams. Thus, we naturally expect

\footnotetext{
${ }^{3}$ For small values of $\lambda,|\lambda| \lesssim \mathcal{O}(4 \pi)$, the mass squared of the Polonyi field is dominated by calculable one-loop corrections and $m_{s}^{2}$ is shown to be positive [10].

${ }^{4}$ Here, we are assuming that $\lambda_{i j} \sim \lambda$.
} 
that the linear term is at least of order $10^{-2}$, i.e. $|c| \gtrsim 10^{-2}$. As we see in the following discussion the linear term in the Kähler potential has a serious effect on the dynamics of the Polonyi field during the inflation.

\subsection{Effects of the Hubble parameter during inflation}

Now, let us consider the dynamics of the Polonyi field during the inflation. First, we assume that the Polonyi field is set to the origin $S=0$ at the beginning of the inflation. We do not discuss, here, what physics provides such a desired situation, since it is beyond the scope of this letter. (If the $Q_{i}$ 's are in the thermal bath before the inflation, the Polonyi field $S$ acquires the thermal mass which drives the $S$ to the origin $S=0$. This may be a possible candidate for the physics.)

Once the inflation starts, the effective potential of the Polonyi field and the inflaton $\phi$ are given by, ${ }^{5}$

$$
V=e^{K}\left(K_{\bar{I} J}^{-1}\left(D_{I} W\right)^{\dagger}\left(D_{J} W\right)-3|W|^{2}\right),(I=\phi, S)
$$

where we have assumed the Kähler potential as,

$$
K=|\phi|^{2}+|S|^{2}-c^{*} S-c S^{\dagger}
$$

for simplicity. $D_{I} W$ and $K_{\bar{I} J}$ are defined by

$$
\begin{aligned}
D_{I} W & =\frac{\partial W}{\partial X_{I}}+\frac{\partial K}{\partial X_{I}} W,\left(X_{I}=\phi, S\right), \\
K_{\bar{I} J} & =\frac{\partial^{2} K}{\partial X_{I}^{\dagger} X_{J}},\left(X_{I}=\phi, S\right) .
\end{aligned}
$$

In addition, we also assume that the hidden sector and the inflaton sector is separated in the superpotential $W$ as,

$$
W=W(S)+W(\phi) .
$$

By using $V_{\text {loop }}$ a potential of $S$ given in Eqs. (16) and (19), $V_{\text {inf }}$ a potential of the inflaton which is nearly constant during the inflation, and $K(S)$ a Kähler potential for the Polonyi field $S$, the above scalar potential can be rewritten as,

$$
V \simeq e^{K(S)}\left(V_{\mathrm{inf}}+V_{\text {loop }}(S)\right)
$$

\footnotetext{
${ }^{5}$ We can easily extend the following discussion to inflation models with many fields.
} 
During the inflation, the potential of the Polonyi field is changed from the one at the true vacuum due to the first term, $e^{K(S)} V_{\text {inf }}$. Here, we have neglected $K(\phi)$, since it is irrelevant to our discussion.

As we have mentioned at the end of the previous subsection, we cannot forbid the linear term in the Kähler potential. During the inflation, such a linear term leads to a slope of the Polonyi potential around the true vacuum $S=0$,

$$
V=e^{K(S)} V_{\mathrm{inf}} \simeq-\left(c^{*} S+c S^{*}\right) V_{\mathrm{inf}} .
$$

On the other hand, the Polonyi potential in Eq. (19) is nearly flat for $S \gtrsim \Lambda / \lambda$ with a height of

$$
V(S)=V(S \gg \Lambda)=\xi\left|\lambda\left(\frac{\Lambda}{4 \pi}\right)^{2}\right|^{2}=3 \xi m_{3 / 2}^{2},
$$

where, $\xi$ is a numerical constant at most of order one. Thus, if the linear term in Eq. (28) is large, the potential minimum is shifted from the origin $S=0$.

To investigate the behavior of $S$ during the inflation more closely, we approximate the above Polonyi potential Eq. (16) and (29) by,

$$
V_{\text {loop }}(S)= \begin{cases}m_{s}^{2}|S|^{2}, & \left(|S| \leq\left|S_{*}\right|\right) \\ 3 \xi m_{3 / 2}^{2}, & \left(|S|>\left|S_{*}\right|\right)\end{cases}
$$

Here, $m_{s}$ is the Polonyi mass given in Eq. (17) and $S_{*}$ is a field value where higher order terms of the effective Kähler potential in Eq. (15) become important. In the following analysis, we take

$$
S_{*}=\eta^{\prime} \frac{\Lambda}{\lambda}
$$

with $\eta^{\prime}=\mathcal{O}(1)$.

Under the above approximation, we find that the true minimum $(S=0)$ is substantially shifted unless a condition,

$$
\left(c^{\dagger} S_{*}+c S_{*}^{\dagger}\right) V_{\mathrm{inf}} \lesssim 3 \xi m_{3 / 2}^{2},
$$

is satisfied. By using Eqs. (18) and (31), this condition can be expressed as an upper bound on the Hubble parameter $H_{\text {inf }}$ during the inflation,

$$
H_{\text {inf }} \lesssim 10^{8} \mathrm{GeV} \times\left(\frac{m_{3 / 2}}{\mathrm{TeV}}\right)^{3 / 4}\left(\frac{10^{16} \mathrm{GeV}}{|c|}\right)^{1 / 2}\left(\frac{\lambda}{4 \pi}\right)^{3 / 4}\left(\xi \eta^{\prime-1}\right)^{1 / 2},
$$



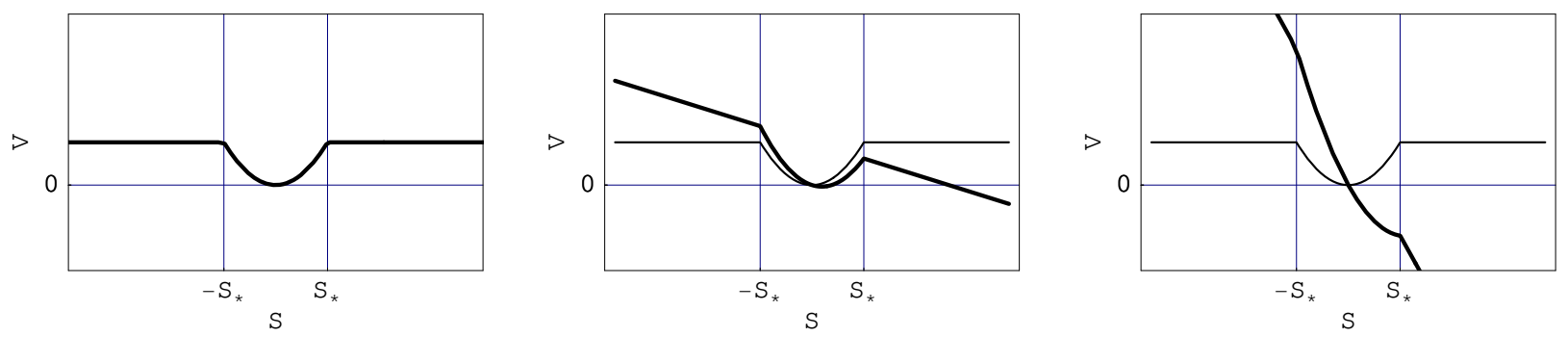

Figure 1: Schematic plots of the Polonyi potential during the inflation. Here, we assume that $\lambda=4 \pi, \xi=\eta^{\prime}=1, c \simeq M_{G}$ and $m_{3 / 2}=1 \mathrm{TeV}$. The potentials correspond to $H_{\mathrm{inf}}=0$, $5 \times 10^{6} \mathrm{GeV}, 10^{8} \mathrm{GeV}$ from left to right, respectively.

where we also used the relation $3 H_{\mathrm{inf}}^{2}=V_{\mathrm{inf}}{ }^{6}$ Figure. 1 is a schematic figure of the Polonyi potential around $S=0$ for various values of $H_{\mathrm{inf}}$. The figure shows that the minimum at $S=0$ is substantially shifted from the origin when the above condition is violated.

Notice that we have derived an upper bound on the Hubble parameter Eq. (33) based on a specific model of the dynamical SUSY-breaking. However, by using a naive dimensional counting 8], we can approximate Polonyi potentials by Eq. (30) with $m_{s} \lesssim \Lambda$ and $S_{*} \gtrsim \Lambda / 4 \pi$ for any effective O'Raifeartaigh models where flat directions are lifted by the quantum corrections. Thus, we consider that the above upper bound on the Hubble parameter is a quite generic result for any effective O'Raifeartaigh models.

Once the minimum at $S=0$ disappears during the inflation, $S$ rolls down to $c$ immediately. In such a case, we suffer from the recurrence of the Polonyi problem, since $S$ is fixed at $S \simeq c$ until the Hubble parameter becomes very small. As we see in the next section, the spilled Polonyi field causes a gravitino overproduction problem for $|c| \gtrsim 10^{-2}$, if $T_{R} \gtrsim 1-100 \mathrm{GeV}$ (see Eq. (44)). Thus, for $|c| \gtrsim 10^{-2}$, the above upper bound on the Hubble parameter, $H_{\text {inf }} \lesssim 10^{8} \mathrm{GeV}$, is a necessary condition for the inflation in the gravity-mediation models, unless the reheating temperature is extremely low, $T_{R} \lesssim 100 \mathrm{GeV} .{ }^{7}$

This result shows that the SUSY chaotic inflation [13] (where typical Hubble parameter is $\left.H_{\mathrm{inf}} \simeq 10^{14} \mathrm{GeV}\right)$ and the SUSY topological inflation [14] $\left(H_{\mathrm{inf}} \simeq 10^{11-14} \mathrm{GeV}\right)$ are disfavored. The SUSY hybrid inflations are also disfavored since the typical Hubble parameters are $H_{\text {inf }} \simeq$ $10^{13-15} \mathrm{GeV}[15]$.

\footnotetext{
${ }^{6}$ We assume inflation models with $F$-term potentials in this letter. However, if we consider $D$-term inflation models, it depends on details of the models if we may evade the constraint Eq. (33).

${ }^{7}$ For the leptogenesis [1] to work, we need a reheating temperature $T_{R}$ higher than the critical temperature for the electroweak phase transition [11, 12. Besides, it seems rather difficult for the inflation to achieve the low reheating temperature such as $T_{R} \ll 10^{6} \mathrm{GeV}$.
} 
On the contrary, among inflation models constructed in SUGRA, a new inflation model in [16, is one of the most attractive candidates. The model has a flat inflaton potential,

$$
V(\varphi) \simeq v^{4}-\frac{k}{2} v^{4} \varphi^{2}-\frac{g}{2^{\frac{n}{2}-1}} v^{2} \varphi^{n}+\frac{g^{2}}{2^{n}} \varphi^{2 n},(n \geq 3)
$$

where $v$ is the energy scale of the inflation, $g$ the coupling constant in the superpotential, and $k$ is the quartic coupling constant in the Kähler potential. ¿From the COBE normalization, the inflation scales are determined for $k \lesssim 10^{-2}$ [17,

$$
\begin{aligned}
& H_{\mathrm{inf}}=\text { undetermined by the COBE normalization, }(n=3), \\
& H_{\mathrm{inf}} \simeq 10^{5.4} \mathrm{GeV} \times \frac{1}{g},(n=4), \\
& H_{\mathrm{inf}} \simeq 10^{8.6} \mathrm{GeV} \times \frac{1}{g^{1 / 2}},(n=5), \\
& H_{\mathrm{inf}} \simeq 10^{9.9} \mathrm{GeV} \times \frac{1}{g^{1 / 3}},(n=6),
\end{aligned}
$$

and $H_{\text {inf }}$ increases for larger $n$. Thus, we find that the new inflation model with $n \gtrsim 5$ is disfavored. Interestingly, the favored new inflation model of $n=4$ predicts the spectral index $n_{s} \simeq 0.94-0.95\left[18\right.$ which is well consistent with the recent WMAP result, $n_{s}=0.951_{-0.019}^{+0.015}$ (68\%C.L.) [19]. ${ }^{8}$

\section{Fate of spilled Polonyi field}

As we have seen in the previous section, if the Hubble parameter is too large and it does not satisfy the condition Eq. (133), the minimum of the Polonyi potential is shifted away from the origin. Then, the Polonyi field falls to the minimum of the potential, $S \simeq c$, during the inflation. In this section, we consider the Polonyi problem for such a case.

In general, there are many local minimal points of the Polonyi potential $V(S)$ around $S=\mathcal{O}\left(M_{G}\right)$, and $S$ has a mass of the order of the gravitino mass at each minimal points. On the contrary, the curvature of the Polonyi potential Eq. (19) at $S \simeq c$ is given by,

$$
V^{\prime \prime}(S) \simeq-\frac{\lambda\left(\mu_{c}\right)^{2}}{4 \pi^{2}|c|^{2}}\left|\lambda\left(\frac{\Lambda}{4 \pi}\right)^{2}\right|^{2} \simeq-\frac{3 \lambda\left(\mu_{c}\right)^{2} m_{3 / 2}^{2}}{4 \pi^{2}|c|^{2}}
$$

with $\mu_{c} \simeq \lambda c$, which is at most the gravitino mass when $c$ is close to $M_{G}$. Hence, for $c \simeq M_{G}$, we do not expect that the Polonyi field returns to $S=0$ after the inflation, since the attractive

\footnotetext{
${ }^{8}$ The detailed analysis on the new inflation model of $n=3$ will be discussed elsewhere [17].
} 
force towards the origin is weaker than those towards the minimal points at $S=\mathcal{O}\left(M_{G}\right)$. In this case, the Polonyi field is attracted to one of the local minimal points at $S=\mathcal{O}\left(M_{G}\right)$ and starts to oscillate around the local minimal point when the Hubble parameter becomes at $H \simeq m_{3 / 2}$ after the inflation. Since the typical distance between the point $S \simeq c$ and the local minimal points at $S=\mathcal{O}\left(M_{G}\right)$ is of the order of the Planck scale, such a late time coherent oscillation causes nothing but the original Polonyi problem [1]. Therefore, once the Polonyi field is spilled out of the $S=0$ minimum, we again suffer from the original Polonyi problem for $c \simeq M_{G}$.

On the other hand, if the linear term is somewhat smaller than the Planck scale, the Polonyi field is attracted to $S=0$ after the inflation, since the curvature of Eq. (39) exceeds the gravitino mass. Then, the Polonyi field starts to oscillate around its true minimum $S=0$ from $S \simeq c$ when the Hubble parameter $H$ falls to $H_{\mathrm{osc}} \simeq \lambda\left(\mu_{c}\right) m_{3 / 2} / c,{ }^{9}$ and it eventually decays dominantly into a pair of gravitinos (see section 2.).

After the whole reheating process, the yield of the gravitino is given by,

$$
Y_{3 / 2}=\frac{n_{3 / 2}}{s} \gtrsim 2 \frac{3 T_{R}}{4 m_{\phi}} \frac{n_{s}}{n_{\phi}} B_{R}
$$

where $n_{s}$ and $n_{\phi}$ denote the number densities of the Polonyi field and the inflaton at $H_{\text {osc }}$. To estimate the yield of the gravitino conservatively, we assume that the Polonyi field decays into a pair of gravitinos with its mass in Eq. (17) immediately after it starts to oscillate around $S=0$. Then, the number density of the Polonyi field and the inflaton at $H_{\mathrm{osc}}$ are given by,

$$
\begin{aligned}
& n_{s} \simeq \frac{\xi}{m_{s}}\left|\lambda\left(\frac{\Lambda}{4 \pi}\right)^{2}\right|^{2} \simeq \frac{3 \xi m_{3 / 2}^{2}}{m_{s}} \\
& n_{\phi} \simeq \frac{\rho_{\phi}}{m_{\phi}} \simeq \frac{3 H_{\mathrm{osc}}^{2}}{m_{\phi}}
\end{aligned}
$$

By using these number densities, we obtain the yield,

$$
Y_{3 / 2} \gtrsim 2 \frac{3 T_{R}}{4 m_{s}} \xi \lambda\left(\mu_{c}\right)^{-2}|c|^{2} B_{R}
$$

Hence, the BBN constraint [2] in Eq. (8) requires,

$$
T_{R} \lesssim 1-100 \mathrm{GeV}\left(\frac{\lambda}{4 \pi}\right)^{5 / 2}\left(\frac{m_{3 / 2}}{1 \mathrm{TeV}}\right)^{1 / 2}\left(\frac{10^{16} \mathrm{GeV}}{|c|}\right)^{2}\left(\xi B_{R}\right)^{-1} \eta^{1 / 2} \lambda\left(\mu_{c}\right)^{2} .
$$

\footnotetext{
${ }^{9}$ We confine ourselves to the inflation model with the reheating temperature $T_{R} \lesssim 10^{6-8}$ GeV, since otherwise we have the gravitino overproduction problem from the scattering process of the thermal background after the inflation [2]. For such reheating temperature, we can safely neglect thermal effects to the Polonyi potential from $Q_{i}$ plasma.
} 
Thus, for $|c| \gtrsim 10^{-2}$, we cannot avoid the gravitino overproduction problem even if the spilled Polonyi field returns to $S=0$ after the inflation, unless the reheating temperature is extremely low. ${ }^{10}$

Notice that the above upper bound on $T_{R}$ is conservative since we have used $m_{s}$ in Eq. (17) to estimate the decay rate of the Polonyi field (Eq. (2) ) and the number density of the Polonyi field (Eq. (41)). Since the amplitude of the Polonyi field is much larger than $\Lambda / \lambda$ at the beginning of the oscillation, the effective mass of the Polonyi field is smaller than $m_{s}$. Thus, one may consider that the decay rate of the Polonyi field is smaller and $n_{s}$ is larger, which leads to a larger number density of the gravitinos. However, even if the effective mass of the Polonyi field is much smaller than $m_{s}$, it behaves as a particle with mass $m_{s}$ during a time period of $1 / m_{s}$ in each oscillation. Thus, the effective decay rate of the Polonyi field (with mass $m_{s}$ ) at the beginning of the coherent oscillation is given by,

$$
\Gamma_{s}^{\mathrm{eff}} \simeq \frac{H_{\mathrm{ocs}}}{m_{s}} \Gamma_{s}
$$

As we see from Eqs. (2) and (17), $\Gamma_{s}$ is close to $m_{s} \simeq \Lambda$ for $\lambda \simeq 4 \pi$, and hence, the effective decay rate is comparable to the Hubble parameter at the beginning of oscillation, $H_{\mathrm{osc}}$. Thus, the Polonyi field effectively decays with a mass $m_{s}$, immediately after it starts to oscillate, and the number density of the Polonyi field which results in the gravitino number density is roughly given by dividing the energy density in Eq. (30) by $m_{s}$ (see Eq. (411)). Therefore, we may use safely the above conservative analysis. ${ }^{11}$

Finally, we comment on possible effects of direct couplings between the hidden sector and the inflaton sector in the superpotential. Although such interactions are highly model dependent (charges of the fields, etc.), we at least expect the terms,

$$
W=W(\phi)\left(1+c_{1} S+c_{2} S^{2}+\cdots\right)
$$

since the Polonyi field is completely neutral under any symmetries. Such terms, in general, increase the number of the minimal points around $S=\mathcal{O}\left(M_{G}\right)$, and hence, the above problems are not improved.

\footnotetext{
${ }^{10}$ Again, we consider that the above upper bound on $T_{R}$ is a quite generic result for effective O'Raifeartaigh models.

${ }^{11}$ In the model we have considered in section 3 , there is a non-anomalous approximate $R$ symmetry. Then, the $Q$-ball and anti- $Q$-ball [20] can be formed after $S$ starts to oscillate around $S=0$. In this case, as long as the annihilation of $Q$-balls can be neglected, the $Q$-ball has a long lifetime, which increases the resultant gravitino abundance. Thus, the upper bound on $T_{R}$ can be much severer than Eq. (44) for the dynamical SUSY breaking model in section 3. We thank F. Takahashi for pointing out this.
} 


\section{Conclusions}

In this letter, we have considered a solution to the Polonyi problem by assuming dynamical SUSY breaking. We have found that even for dynamical SUSY breaking models, the linear term $c$ of the Polonyi field in the Kähler potential may bring us back to the Polonyi problem or the gravitino overproduction problem. To avoid the problems for the most natural case, $|c| \gtrsim 10^{-2}$, in the gravity-mediation models the inflation in the early universe should have a

very small Hubble parameter, $H_{\text {inf }} \lesssim 10^{8} \mathrm{GeV}$ (Eq. (33)) such as new inflation models, or a very low reheating temperature, $T_{R} \lesssim 10^{2} \mathrm{GeV}$ (Eq. (44) ). ${ }^{12}$ This result is very interesting since the favored new inflation model in SUGRA naturally predicts the spectral index as $n_{s} \simeq 0.94-0.95$, which is very consistent with the recent WMAP observation.

We comment that there is also no theoretical reason to suppress the Kähler interactions such as $K=\kappa|\phi|^{2} S^{2}$. If it exists, the inflaton decay into a pair of gravitinos is enhanced and a stringent constraint on the inflation model is obtained. The recent analysis has shown that the hybrid inflation model is very disfavored [4]. Furthermore, the interactions between the hidden sector and the inflaton sector in the superpotential can enhance the inflaton decay rate into a pair of gravitinos, which may give more stringent constraints. The detailed analysis in the new inflation model [16] including such superpotential interactions will be discussed elsewhere [17].

We should note finally that the Polonyi problem discussed in this letter may not exist in gauge- or anomaly-mediation models for SUSY breaking. This is because the Polonyi field may have charges of some symmetries suppressing the linear term in the Kähler potential or because there is not necessarily present the elementary Polonyi field in those models.

\section{Acknowledgments}

M. I. thanks the Japan Society for the Promotion of Science for financial support. This work is partially supported by Grand-in-Aid Scientific Research (s) 14102004. The work of T.T.Y. has been supported in part by a Humboldt Research Award.

\section{References}

[1] G. D. Coughlan, W. Fischler, E. W. Kolb, S. Raby and G. G. Ross, Phys. Lett. B 131, 59 (1983).

[2] M. Kawasaki, K. Kohri and T. Moroi, Phys. Rev. D 71 (2005) 083502 arXiv:astro-ph/0408426, and references therein.

\footnotetext{
${ }^{12}$ If there is a late-time entropy production, the constraint on the reheating temperature can be weakened.
} 
[3] M. Endo, K. Hamaguchi and F. Takahashi, arXiv:hep-ph/0602061, arXiv:hep-ph/0605091; S. Nakamura and M. Yamaguchi, arXiv:hep-ph/0602081 T. Asaka, S. Nakamura and M. Yamaguchi, arXiv:hep-ph/0604132 M. Dine, R. Kitano, A. Morisse and Y. Shirman, arXiv:hep-ph/0604140.

[4] M. Kawasaki, F. Takahashi and T. T. Yanagida, arXiv:hep-ph/0603265 M. Kawasaki, F. Takahashi and T. T. Yanagida, arXiv:hep-ph/0605297.

[5] K. I. Izawa and T. Yanagida, Prog. Theor. Phys. 94, 1105 (1995) arXiv:hep-ph/9507441.

[6] K. I. Izawa and T. Yanagida, Prog. Theor. Phys. 95, 829 (1996) arXiv:hep-th/9602180; K. A. Intriligator and S. D. Thomas, Nucl. Phys. B 473, 121 (1996) arXiv:hep-th/9603158.

[7] K. A. Intriligator, R. G. Leigh and N. Seiberg, Phys. Rev. D 50, 1092 (1994) arXiv:hep-th/9403198.

[8] M. A. Luty, Phys. Rev. D 57, 1531 (1998) arXiv:hep-ph/9706235; A. G. Cohen, D. B. Kaplan and A. E. Nelson, Phys. Lett. B 412, 301 (1997) arXiv:hep-ph/9706275.

[9] N. Arkani-Hamed and H. Murayama, Phys. Rev. D 57, 6638 (1998) arXiv:hep-th/9705189.

[10] Z. Chacko, M. A. Luty and E. Ponton, JHEP 9812 (1998) 016 arXiv:hep-th/9810253.

[11] M. Fukugita and T. Yanagida, Phys. Lett. B 174, 45 (1986); For a review, W. Buchmuller, R. D. Peccei and T. Yanagida, Ann. Rev. Nucl. Part. Sci. 55, 311 (2005) arXiv:hep-ph/0502169.

[12] M. Ibe, T. Moroi and T. Yanagida, Phys. Lett. B 620, 9 (2005) arXiv:hep-ph/0502074.

[13] A. B. Goncharov and A. D. Linde, Phys. Lett. B 139, 27 (1984); Class. Quant. Grav. 1 (1984) L75; H. Murayama, H. Suzuki, T. Yanagida and J. Yokoyama, Phys. Rev. D 50, 2356 (1994) arXiv:hep-ph/9311326; M. Kawasaki, M. Yamaguchi and T. Yanagida, Phys. Rev. Lett. 85, 3572 (2000) arXiv:hep-ph/0004243.

[14] K. I. Izawa, M. Kawasaki and T. Yanagida, Prog. Theor. Phys. 101, 1129 (1999) arXiv:hep-ph/9810537; M. Kawasaki, N. Sakai, M. Yamaguchi and T. Yanagida, Phys. Rev. D 62, 123507 (2000) arXiv:hep-ph/0005073; M. Kawasaki and M. Yamaguchi, Phys. Rev. D 65, 103518 (2002) arXiv:hep-ph/0112093.

[15] G. R. Dvali, Q. Shafi and R. K. Schaefer, Phys. Rev. Lett. 73, 1886 (1994) arXiv:hep-ph/9406319; E. D. Stewart, Phys. Lett. B 345, 414 (1995) arXiv:astro-ph/9407040; G. Lazarides and C. Panagiotakopoulos, Phys. Rev. D 52, 559 (1995) arXiv:hep-ph/9506325; A. D. Linde and A. Riotto, Phys. Rev. D 56, 1841 (1997) arXiv:hep-ph/9703209.

[16] K. Kumekawa, T. Moroi and T. Yanagida, Prog. Theor. Phys. 92, 437 (1994) arXiv:hep-ph/9405337; K. I. Izawa and T. Yanagida, Phys. Lett. B 393, 331 (1997) arXiv:hep-ph/9608359 ; T. Asaka, K. Hamaguchi, M. Kawasaki and T. Yanagida, Phys. Rev. D 61, 083512 (2000) arXiv:hep-ph/9907559; V. N. Senoguz and Q. Shafi, Phys. Lett. B 596, 8 (2004) arXiv:hep-ph/0403294.

[17] M. Ibe and Y. Shinbara, in preparation.

[18] M. Ibe, K. I. Izawa, Y. Shinbara and T. T. Yanagida, Phys. Lett. B 637, 21 (2006) arXiv:hep-ph/0602192 ; K. I. Izawa, Phys. Lett. B 576, 1 (2003) arXiv:hep-ph/0305286.

[19] D. N. Spergel et al., arXiv:astro-ph/0603449.

[20] S. R. Coleman, Nucl. Phys. B 262, 263 (1985) [Erratum-ibid. B 269, 744 (1986)]. 